\title{
Visceral leishmaniasis: rapid response to AmBisome treatment
}

\author{
O P Smith, I M Hann, H Cox, V Novelli
}

\begin{abstract}
There appears to be an increase in imported cases of visceral leishmaniasis in Northern Europe; many are children infected on holiday in the Mediterranean. Making the diagnosis in young children can be difficult especially when an adequate travel history is not obtained at presentation. Two infants with visceral leishmaniasis are presented who were initially felt to have alternative diagnoses and who subsequently responded dramatically to a short course of liposomal amphotericin B (AmBisome). (Arch Dis Child 1995; 73: 157-159)
\end{abstract}

Keywords: leishmaniasis, AmBisome, differential diagnosis.

Visceral leishmaniasis or kala-azar (Hindu for black fever) affects 400000 people annually. ${ }^{1}$ Other names for the condition include sirkari disease, burduvan fever, and Dumdum fever. While the majority of these cases occur in India, Africa, the Mediterranean basin, South America and China, there appears to be an increase in the number of imported cases in Northern Europe. A significant number of these are children who have become infected with Leishmania infantum while on holiday in the Mediterranean. The typical presentation of progressive splenomegaly, hepatomegaly, anaemia, wasting, and fever is seen in both children and adults and the diagnosis is usually confirmed from bone marrow culture and bone marrow aspirate/trephine histology in suspected cases. Making the diagnosis of visceral leishmaniasis, however, can be difficult if the condition is not considered and when a proper travel history is not taken. We report two children with visceral leishmaniasis in whom the diagnosis was delayed due to alternative diagnoses being considered, but who both nevertheless responded dramatically to a short course of liposomal amphotericin B (AmBisome; Vestar).

\section{Case reports}

CASE 1

A 12 month old girl was referred to our hospital with a possible diagnosis of acute lymphoblastic leukaemia. During the previous eight weeks she had developed intermittent fevers with associated ear and chest infections. She was one of twins born at 37 weeks to non-consanguineous parents and was fully vaccinated for age. Examination showed her to be febrile $\left(38.8^{\circ} \mathrm{C}\right)$, anaemic with hepatomegaly $(1 \mathrm{~cm})$ and splenomegaly $(3 \mathrm{~cm})$ but no peripheral lymphadenopathy. The haematological findings included a haemoglobin concentration of $86 \mathrm{~g} / 1$ with normochromic, normocytic indices, white cell count $7 \cdot 4 \times 10^{9} / 1$ (neutrophils $1.6 \times 10^{9} /$, lymphocytes $5.8 \times 10^{9} / 1$ ), platelets $111 \times 10^{9} /$, and normal coagulation profile. Morphological examination of the blood revealed abnormal lymphoid cells compatible with lymphoblasts and a provisional diagnosis of acute lymphoblastic leukaemia was made. Erythrocyte sedimentation rate was raised at 43 $\mathrm{mm} /$ hour but renal and liver function were normal.

On admission to our hospital bone marrow aspirates and trephine biopsies from the posterior superior iliac crests were carried out. Both tissue histologies showed an infiltrate of Leishman-Donovan bodies (amastigotes) to be present within the macrophage compartment (figure). There was no evidence of leukaemia on morphological or by immunophenotypic criteria. A diagnosis of visceral leishmaniasis was made and the patient was started on AmBisome ( $3 \mathrm{mg} / \mathrm{kg} /$ day intravenously for 10 days). Retrospective questioning revealed that the family had been to Nice, southern France some two months before the child became unwell. Four days into treatment she became afebrile with normal blood count and disappearance of the hepatosplenomegaly. Her leishmania serology was: direct agglutination test (DAT) positive to $1: 1600$ and indirect immunofluorescence antibody test (IFAT) negative. A repeat bone marrow trephine three weeks later showed no evidence of leishmaniasis. Nine months after AmBisome treatment she remains well.

\section{CASE 2}

A 14 month old boy was admitted to our hospital for further investigation of recurrent

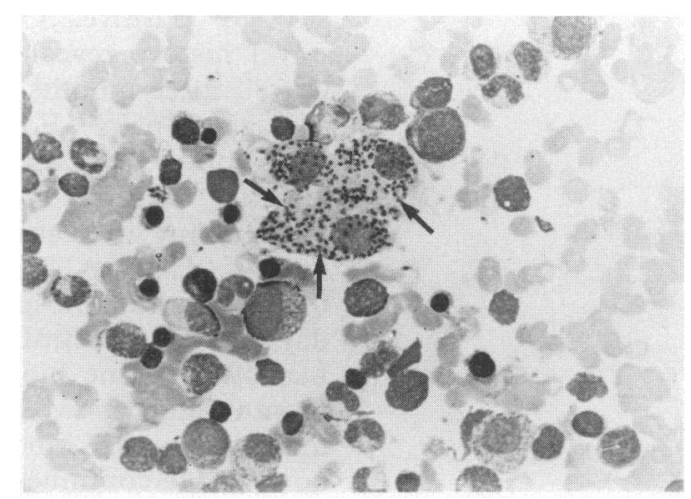

Amastigotes (Leishman-Donovan bodies) present inside macrophages in bone marrow trephine in case 1. 
fevers of six weeks' duration, weight loss, upper respiratory tract infections, massive hepatosplenomegaly, shotty peripheral lymphadenopathy, and pancytopenia. The blood film at presentation did not show any abnormal cells, in particular there was no evidence of leukaemia. A bone marrow aspirate carried out at that stage showed a normocellular marrow for age with active granulopoiesis and megakaryocytopoiesis and some mild dysplastic change in the erythroid series. There was no evidence of leukaemia or indeed any infiltrative process present. He was the first child of unrelated parents and was fully up to date with his immunisations.

On examination at our hospital he was febrile $\left(39^{\circ} \mathrm{C}\right)$, anaemic with hepatomegaly $(7 \mathrm{~cm})$, splenomegaly $(8 \mathrm{~cm})$, and shotty axillary and inguinal lymphadenopathy. Initial investigations included a full blood count with a haemoglobin concentration of $90 \mathrm{~g} / \mathrm{l}$, white cell count $2.4 \times 10^{9} / 1$ (neutrophils $0 \cdot 2 \times 10^{9} / 1$, lymphocytes $\left.2 \cdot 2 \times 10^{9} / 1\right)$, platelets $44 \times 10^{9} / 1$. White cell, red cell, and platelet morphology were normal on blood smear examination. Coagulation testing showed a mild coagulopathy; prothrombin time 16 seconds (control 14 seconds), activated partial thromboplastin time 48 seconds (control 36 seconds), thrombin time 11 seconds (control 10 seconds), fibrinogen $5.3 \mathrm{~g} / \mathrm{l}$. His blood chemistry showed a sodium concentration of $131 \mathrm{mmol} / \mathrm{l}$, potassium $4.8 \mathrm{mmol} / 1$, urea $5.8 \mathrm{mmol} / 1$, creatinine $33 \mu \mathrm{mol} / \mathrm{l}$, calcium $2 \cdot 1 \mathrm{mmol} / \mathrm{l}$, albumin $30 \mathrm{~g} / \mathrm{l}$, aspartate aminotransferase $107 \mathrm{IU} / 1$ (raised), cholesterol $2.4 \mathrm{mmol} / 1$ (normal), triglycerides $3.82 \mathrm{mmol} / \mathrm{l}$ (raised). Ultrasound examination of the abdomen showed gross enlargement of the liver and spleen with normal echogenicity and no focal lesions. Morphological assessment of a repeat bone marrow aspirate showed an active hypercellular marrow with left shifted granulopoiesis, moderate dyserythropoiesis and dysmegakryocytopoiesis, with no evidence of haemophagocytosis, non-malignant infiltration, or leukaemia. During the first week the child required red cell and platelet support and it was felt the most likely diagnosis was haemophagocytic lymphohistiocytosis, despite there being no evidence of haemophagocytosis in the bone marrow. Trephine histology showed an infiltrate of Leishman-Donovan bodies. His leishmania serology was: DAT positive to 1:51200 and leishmania IFAT also strongly positive. Once again retrospective travel history revealed that he had become unwell three months after a family holiday in Malaga, Spain. Four days after starting AmBisome ( $3 \mathrm{mg} / \mathrm{kg} /$ day intravenous) he was afebrile, continued to have hepatosplenomegaly, but was eating and much brighter in himself. He was discharged two days later to his referring hospital to complete his 10 day course of AmBisome. A repeat bone marrow trephine biopsy specimen taken one month later showed no evidence of leishmaniasis and full blood count had returned to normal. He continues to be in remission eight months after completion of AmBisome treatment.

\section{Discussion}

Leishmaniasis is an obligate parasitic infection of macrophages particularly of those occupying the red pulp of the spleen and other reticuloendothelial structures. Establishing the diagnosis of visceral leishmaniasis requires the demonstration of amastigotes from spleen, bone marrow, liver, or lymph nodes. Splenic aspiration cytology is the most sensitive method of diagnosis; however, it is only used in endemic areas. ${ }^{2}$ In sporadic cases the diagnostic method of choice is culture of bone marrow from aspiration and trephine biopsy alone with morphological examination of Giemsa or Leishman stained smears. ${ }^{3}$ Trephine biopsy gives a more quantitative assessment of bone marrow architecture than aspirates, and hence is more likely to reveal the presence of parasites, as was the situation in case 2 . It should be remembered, however, that it may be very difficult to obtain trephines from infants and very young children, especially in institutions not routinely carrying out these procedures. When this arises referral to a specialist centre should be sought. Serodiagnosis is less valuable in individual cases than in epidemiological studies; however, when visceral leishmaniasis is suspected in a patient then serodiagnosis is often very informative.

It is interesting that at the initial interview both sets of parents denied that their children had been to a foreign country but subsequently agreed that they had been to the Mediterranean. This was because they were asked whether or not they had been on an 'exotic' holiday and the parents did not regard the Mediterranean as coming within this category. The other set of parents were asked about 'recent' holidays abroad and thus did not report a visit to Spain three months previously. Clearly, the diagnosis of visceral leishmaniasis would have been reached much sooner if a travel history reaching back to birth and more direct accurate questioning had been pursued.

Until recently the cornerstone of treatment of visceral leishmaniasis has been the pentavalent antimonials, sodium stibogluconate and meglumine antimoniate. While these drugs are efficacious they do have a number of disadvantages, namely that they require intravenous administration usually on an inpatient basis for at least three weeks, cardiac and renal toxicity is not uncommon, and there is now evidence of increasing primary resistance in India and to a lesser extent in Europe. ${ }^{4}$ Amphotericin B has selective toxicity for $L$ infantum because of its increased affinity for ergosterol precursors in the cell membrane of the protozoan over that of cholesterol in mammalian cell membrane. ${ }^{5}$ Its use is also limited by serious side effects and poor tolerance, although in a recently reported trial from India a lower dose of amphotericin B $(0.5 \mathrm{mg} / \mathrm{kg})$ was shown to be efficacious. ${ }^{6}$ Its liposomal counterpart (AmBisome), has been effective in the primary treatment of visceral leishmaniasis and antimonial resistant visceral leishmaniasis with no toxicity and requires administration for 10 days only in the dosage outlined above. The reason for this improved 
therapeutic index lies in the fact that macrophages in reticuloendothelial structures remove particulate and vesicular drug carriers from the circulation and it is this site specifically targeted by liposomal amphotericin B that makes it also more efficacious than its native compound. At the time of writing the cost of the liposomal preparation precludes its use in endemic areas where it is needed most. It may be possible to circumvent this financial problem by reducing the duration of AmBisome treatment and give the drug solely on an outpatient basis and thus reduce the cost to that of the current antimonials regimens. Trials in progress with AmBisome in Africa,
Brazil, and Europe should be helpful in this respect.

1 Marsden PD. Selective primary health care: strategies for control of disease in the developing world. Rev Infect Dis 1984; 6: 736-44.

2 Kager PA, Rees PH. Splenic aspiration: review of the literature. Trop Geogr Med 1983; 35: 111-24.

3 Siddig M, Ghalib H, Shillington DC, Petersen EA. Visceral leishmaniasis in the Sudan: comparative parasitological methods of diagnosis. Roy Soc Med Hyg 1988; 82: 66-8.

4 Davidson RN, Di Martino L, Gradoni L, et al. Liposomal amphotericin B (AmBisome) in Mediterranean visceral amphotericin B (AmBisome) in Mediterranean visceral 5 Gradoni L, Davidson RN, Orsini S, Betto P, Giambenedetti M. Activity of liposomal amphotericin B (AmBisome) $M$. Activity of liposomal amphotericin $B$ (AmBisome)
against Leishmania infantum and tissue distribution in mice. Fournal of Drug Targeting 1993; 1: 311-6.

6 Mishra M, Biswas UK, Jha AM, Khan AB. Amphotericin versus sodium stibogluconate in first-line treatment of Indian kala-azar. Lancet 1994; 334: 1599-600. 